\title{
Trimethoprim sulfamethoxazole drug resistance with co resistance to extended spectrum $\beta$-lactam antibiotics among bacterial isolates from HIV patients
}

\author{
Marimuthu Ragavan Rameshkumar ${ }^{1}$, Nallusamy Vijaykanth, Ramachandran Vignesh², Pachamuthu Balakrishnan², \\ Suniti Solomon ${ }^{2}$, Narasingam Arunagirinathan ${ }^{1 *}$
}

From 2nd International Science Symposium on HIV and Infectious Diseases (HIV SCIENCE 2014)

Chennai, India. 30 January - 1 February 2014

\section{Background}

Trimethoprim-sulfamethoxazole (TMP-SMX) is a broad spectrum antimicrobial agent and also reduces the mortality among adults and children when used as prophylaxis against opportunistic infections in HIV infected patients. Drug resistant to TMP-SMX along with Extended spectrum $\beta$-lactamase (ESBL) production among Enterobacteriaceae is creating major therapeutic problem in clinical settings for treating the bacterial infections among HIV individuals.

\section{Methods}

TMP-SMX drug resistance among the isolates was identified using Kirby-Bauer disc diffusion method and ESBL production by combination disc method (CDM). Cefotaxime $(30 \mu \mathrm{g})$ and cefotaxime/clavulanic acid $(30 \mu \mathrm{g} / 10 \mu \mathrm{g})$ discs were placed $20 \mathrm{~mm}$ apart on the agar surface. Similarly, the ceftazidime $(30 \mu \mathrm{g})$ and ceftazidime/clavulanic acid $(30 \mu \mathrm{g} / 10 \mu \mathrm{g})$ discs were also placed. After incubating overnight at $37^{\circ} \mathrm{C}, \mathrm{a} \geq 5 \mathrm{~mm}$ increase in the zone diameter was interpreted as positive for ESBL production. Statistical analysis was done using SPSS software version 15.0.

\section{Results}

A total of 103(40 Escherichia coli, 15 Klebsiella pneumoniae, 13 Pseudomonas aeruginosa, 10 Klebsiella oxytoca, 8 Proteus mirabilis, 2 Proteus vulgaris, 11 Staphylococcus aureus, 3 Staphylococcus epidermidis and 1 Streptococcus sp.) bacterial strains were isolated from HIV patients. Among these $65(63.10 \% ; p=0.008)$ isolates were resistance to TMPSMX and only $40(38.83 \% ; p=0.023)$ isolates were resistant to extended spectrum $\beta$-lactam antibiotics. Twenty nine ESBL producers from HIV patients were found to be co resistant to TMP-SMX. All ESBL producing isolates showed resistance to ceftazidime and also for ceftazidime/clavulanic acid combination.

\section{Conclusion}

A rapid increase in the use of prophylactic TMP-SMX might be responsible for the TMP-SMX drug resistance among opportunistic bacterial infections in HIV patients.

\section{Authors' details}

${ }^{1} P G$ \& Research Department of Microbiology and Biotechnology, Presidency College (Autonomous), Chennai, India. ${ }^{2}$ YRG Centre for AIDS Research and Education, Chennai, India.

Published: 27 May 2014

doi:10.1186/1471-2334-14-S3-P57

Cite this article as: Rameshkumar et al:: Trimethoprim sulfamethoxazole drug resistance with co resistance to extended spectrum $\beta$-lactam antibiotics among bacterial isolates from HIV patients. BMC Infectious Diseases 2014 14(Suppl 3):P57.

\footnotetext{
* Correspondence: n_arunagiri@yahoo.co.in

${ }^{1} \mathrm{PG} \&$ Research Department of Microbiology and Biotechnology, Presidency College (Autonomous), Chennai, India

Full list of author information is available at the end of the article
} 